\title{
Feasibility and Cost Optimization Study of Osmotic Assisted Reverse Osmosis Process for Brine Management
}

\section{Mona Gulied ${ }^{1}$, Ahmed Al Nouss ${ }^{2}$, Tasneem EIMakki ${ }^{1}$, Fathima Sifani Zavahir ${ }^{1}$, Dong Suk Han ${ }^{1}$}

خامعــة خليفة

HAMAD BIN KHALIFA

UNIVERSITY $\triangle$

Member of Q Qatar Foundation
${ }^{1}$ Center for Advanced Material, Qatar University, PO Box 2713, Doha Qatar

${ }^{2}$ Division of Sustainable Development, College of Science and Engineering, Hamad Bin Khalifa University, Education City, Doha

\section{Problem statement and Motivation}

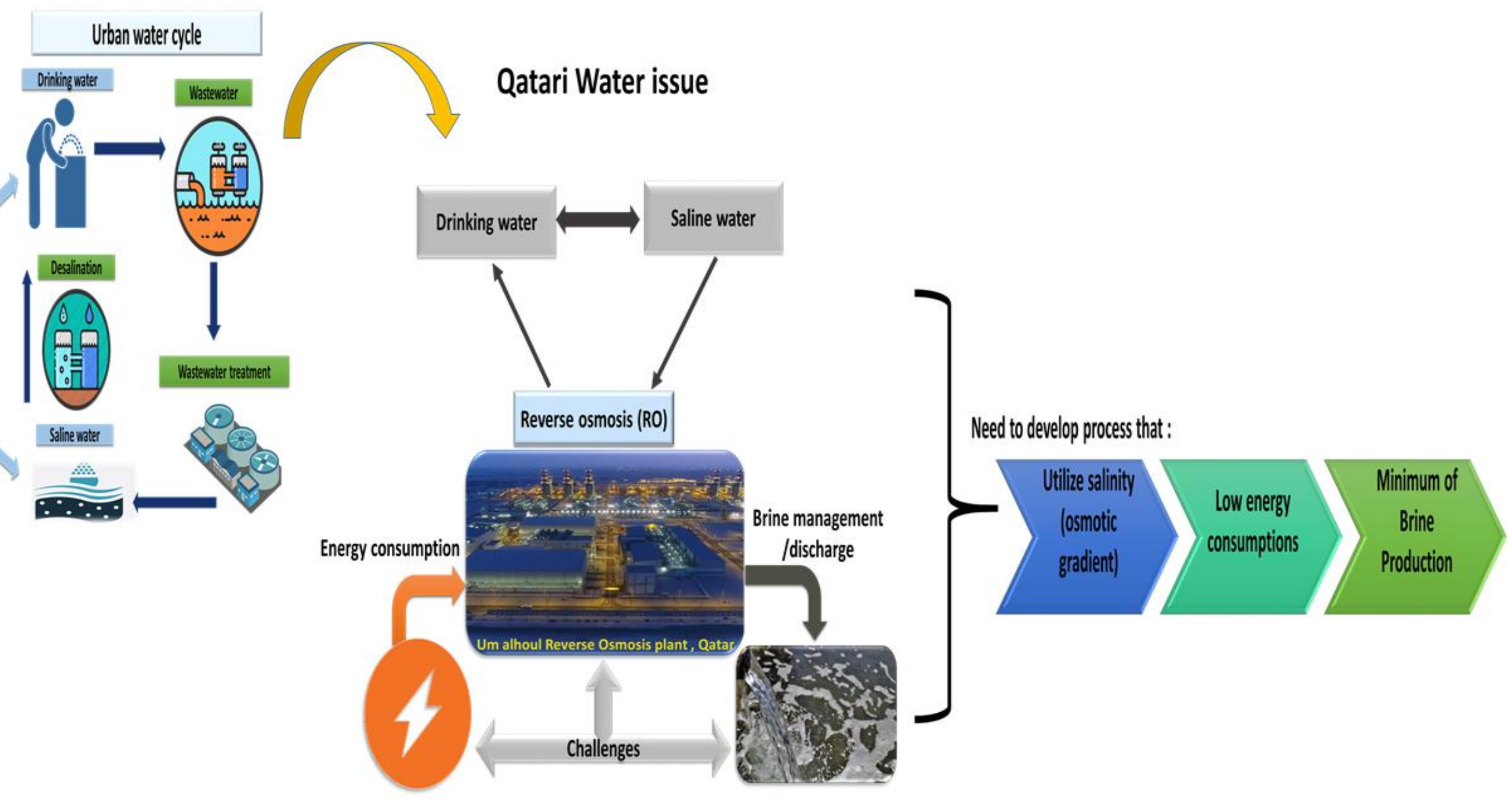

3
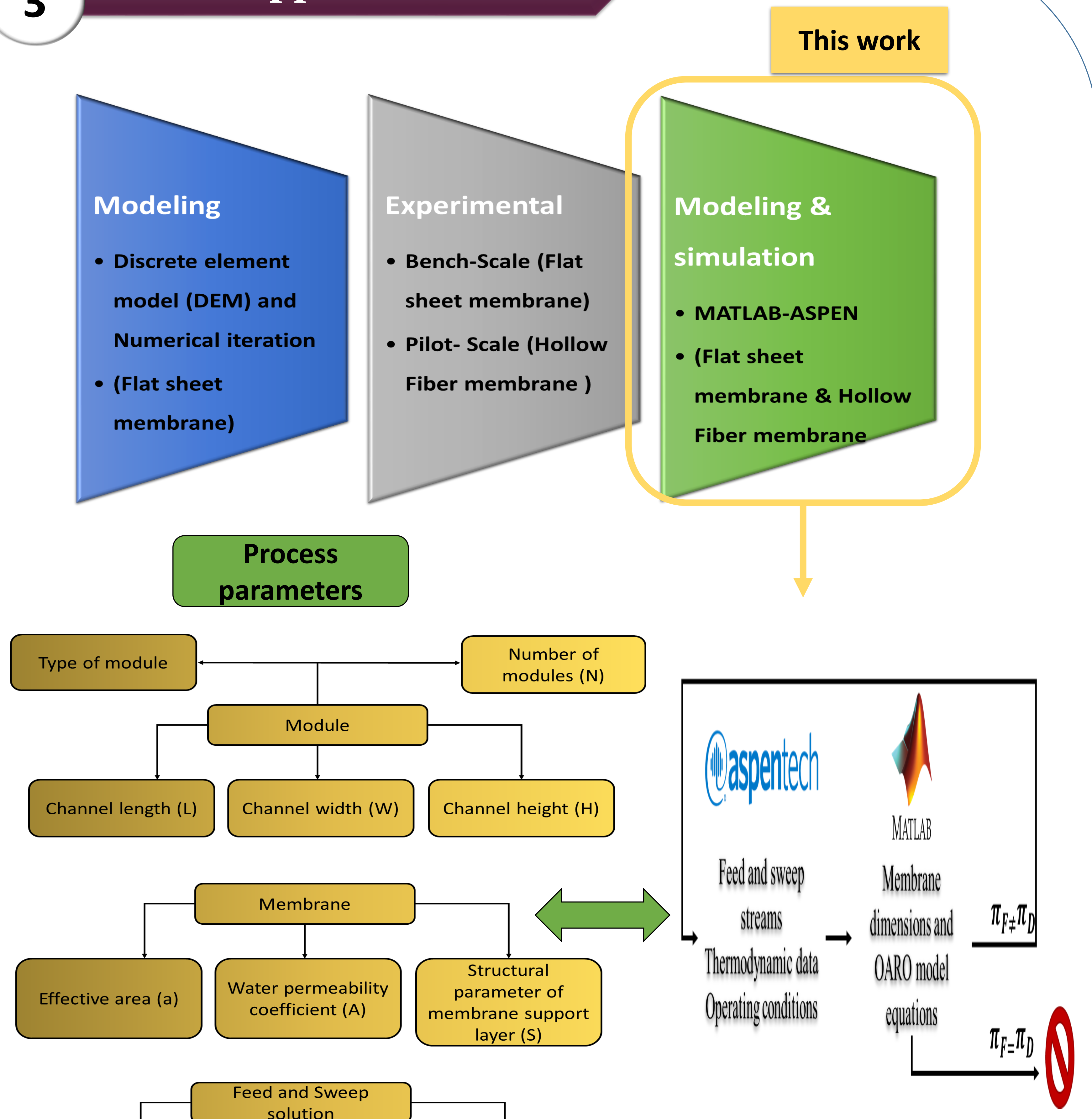

Solute
concentration (C) Flowrate (F) $\begin{gathered}\text { Inlet hydraulic } \\ \text { pressure (P) }\end{gathered}$

5

- Increasing inlet Feed pressure lead to reduction of water recovery\% (\%WR).

- Inlet hydraulic pressure must not exceed 70 bar.

- Increasing membrane active area per module decreases \%WR.

- Increasing \% WR requires more number of stages and energy consumption.

6

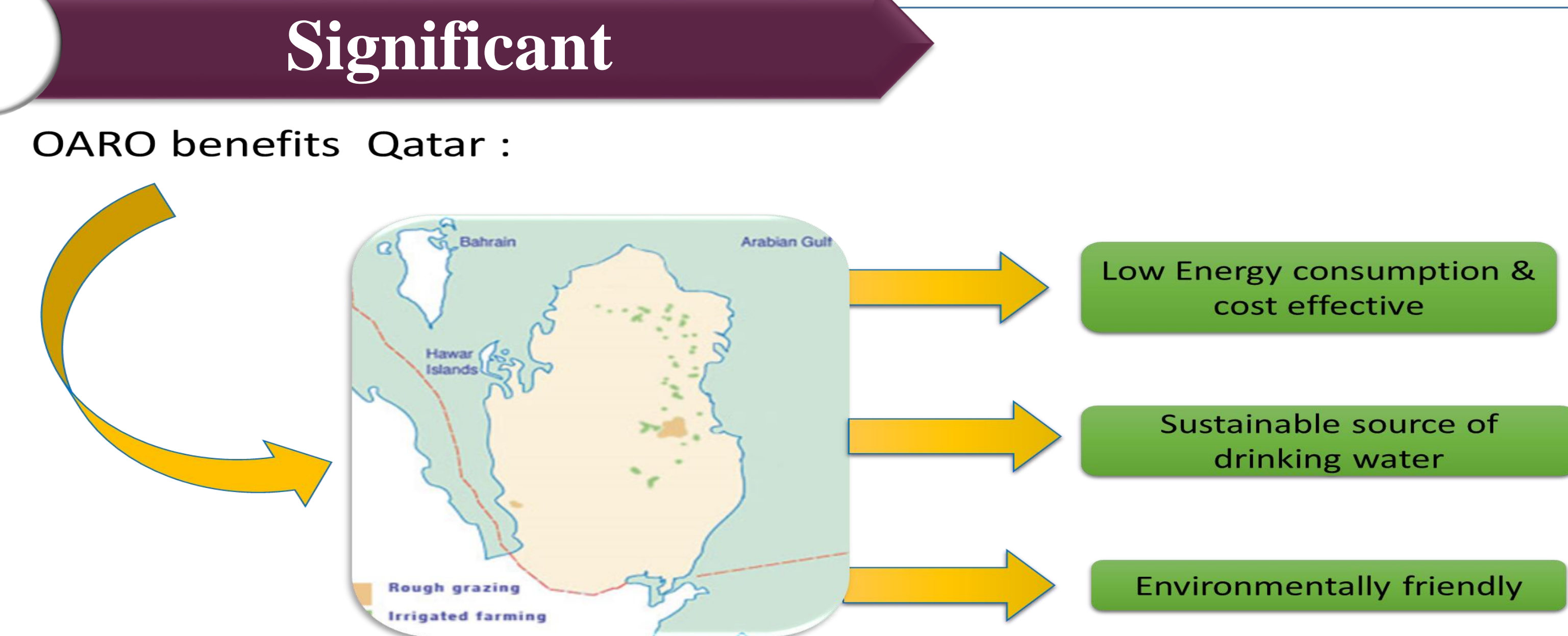

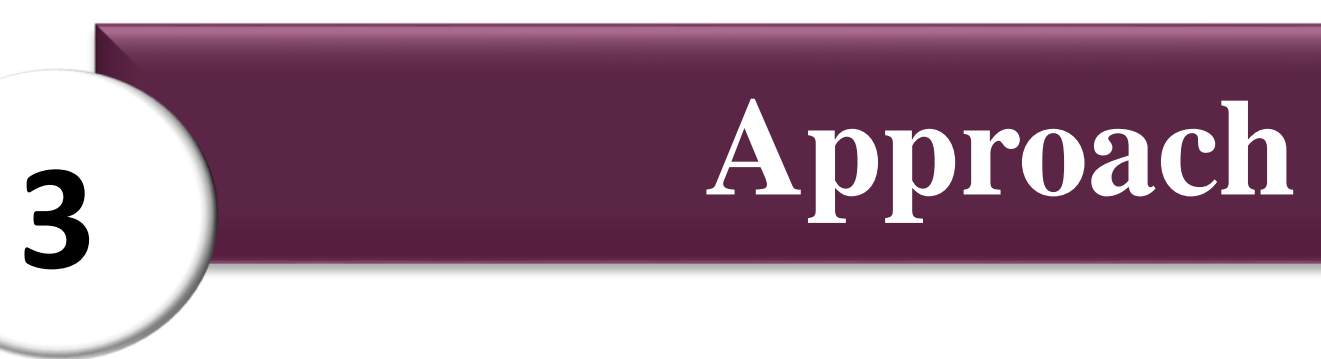

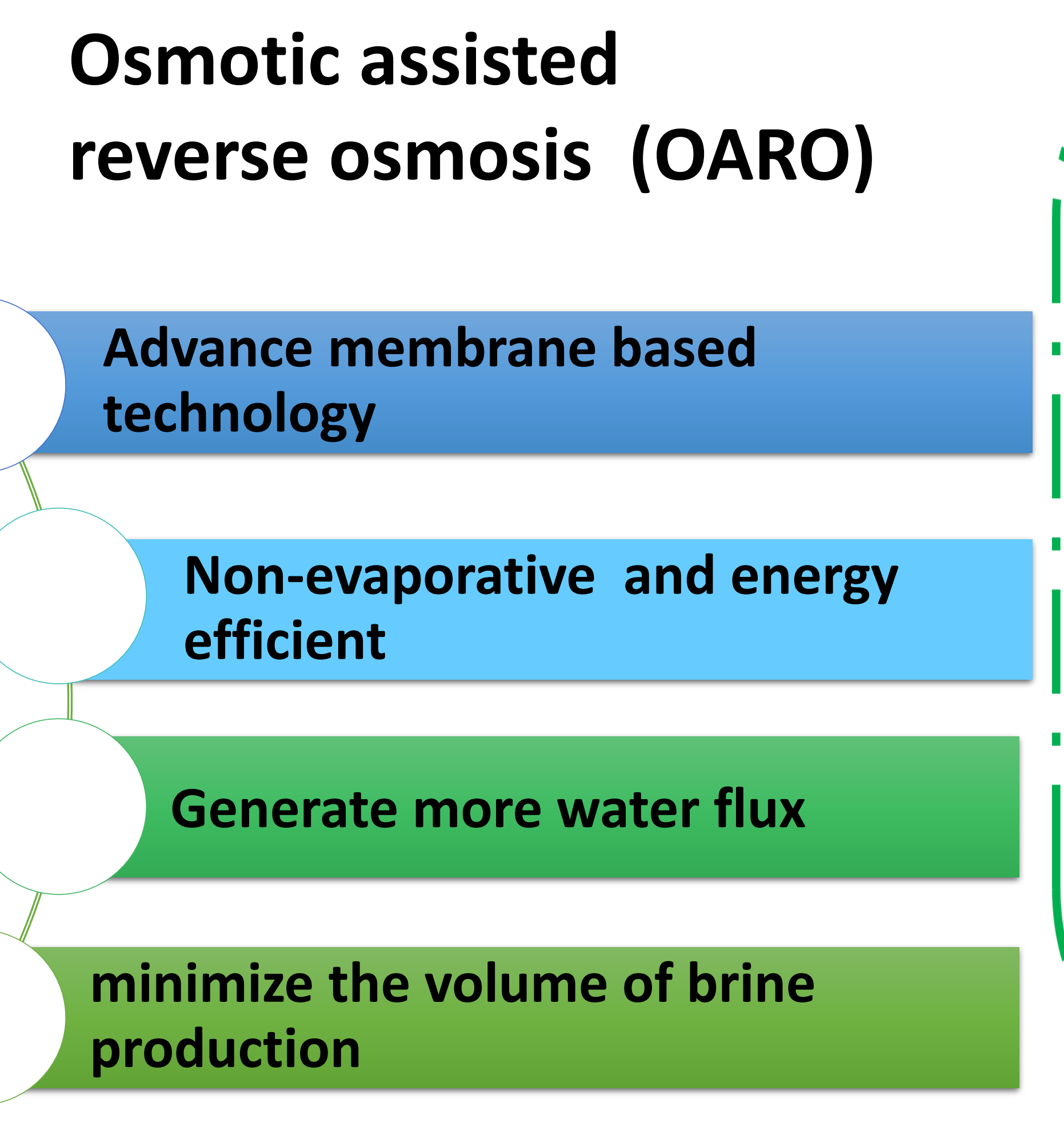

Background and Objectives ( 2

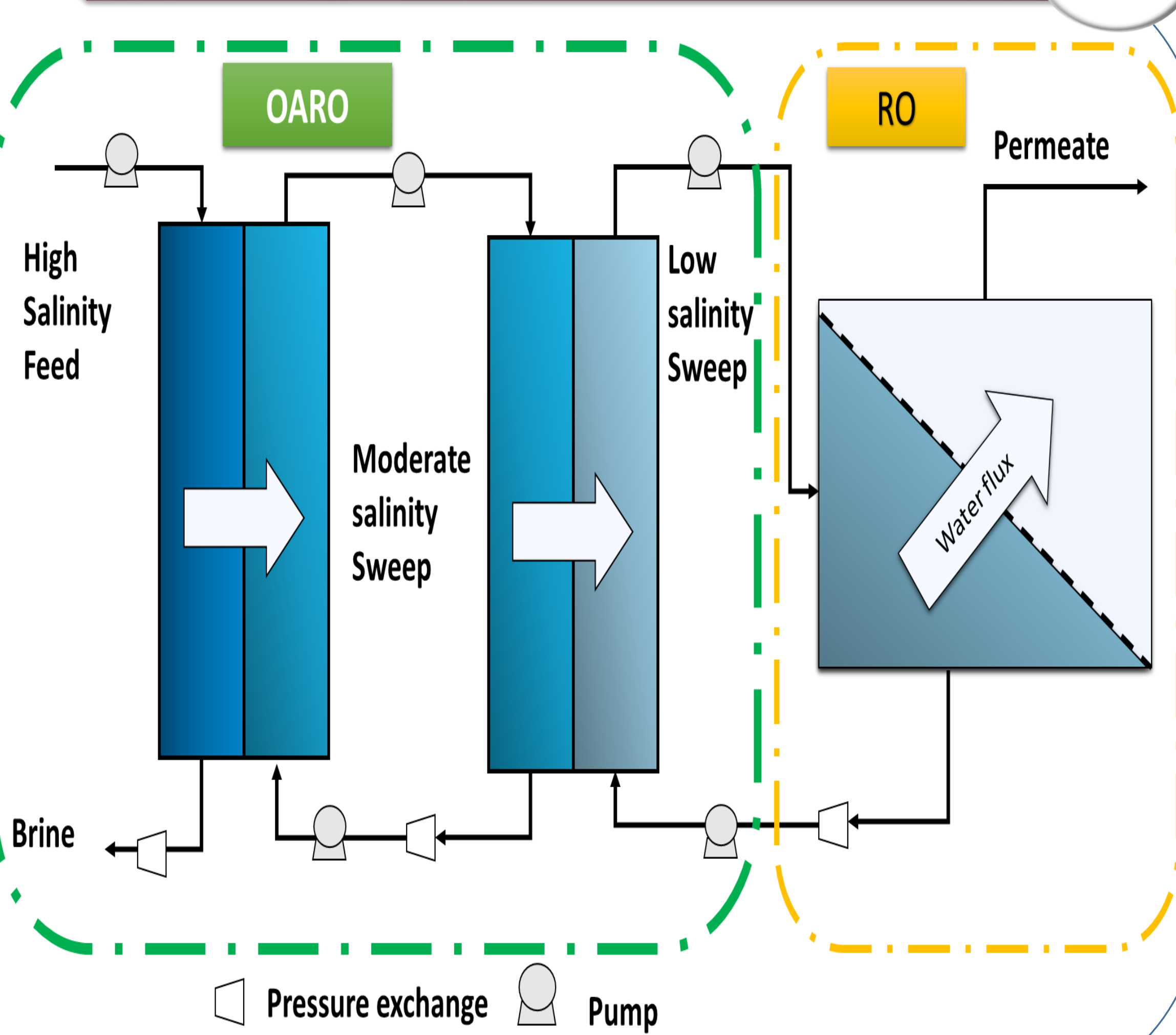

Results \& Discussion 4
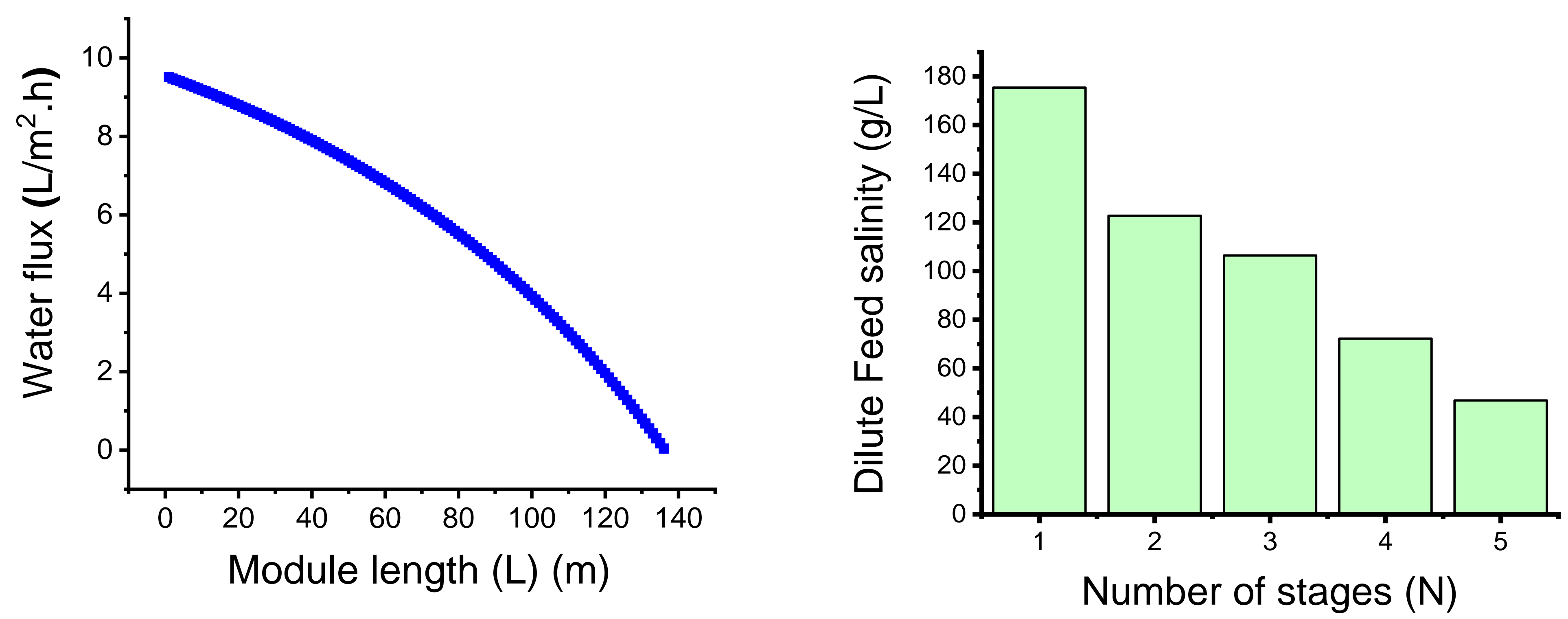

Figure 1: (a) Water flux (b) Diluted feed salinity. Feed Conc. $(175 \mathrm{~g} / \mathrm{L})$, Temperature $\left(25^{\circ} \mathrm{C}\right)$, inlet pressure (65 bar) and flowrate (0.6LPM)

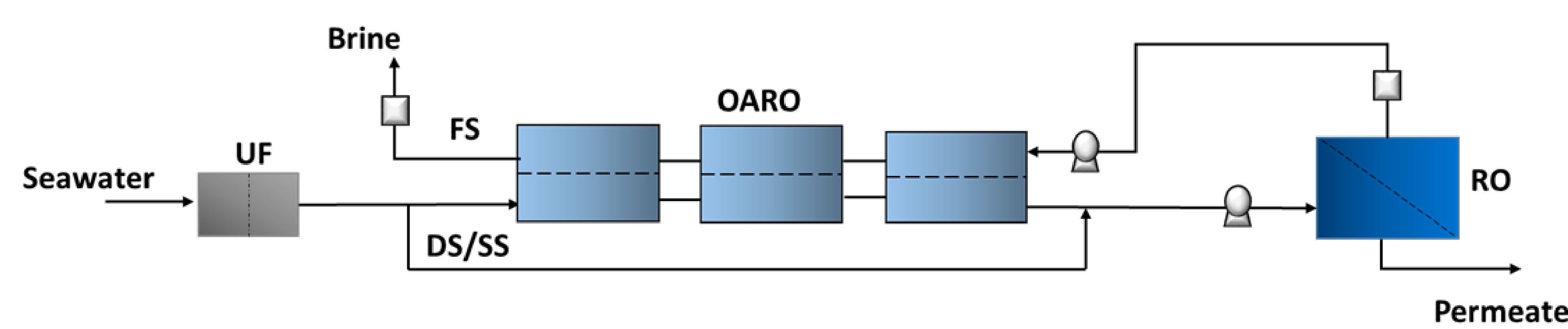

Figure 2: Adapted Configuration flow, Cascading flow. Feed solution (FS), Draw solution (DS) or Sweep solution(SS). [2]

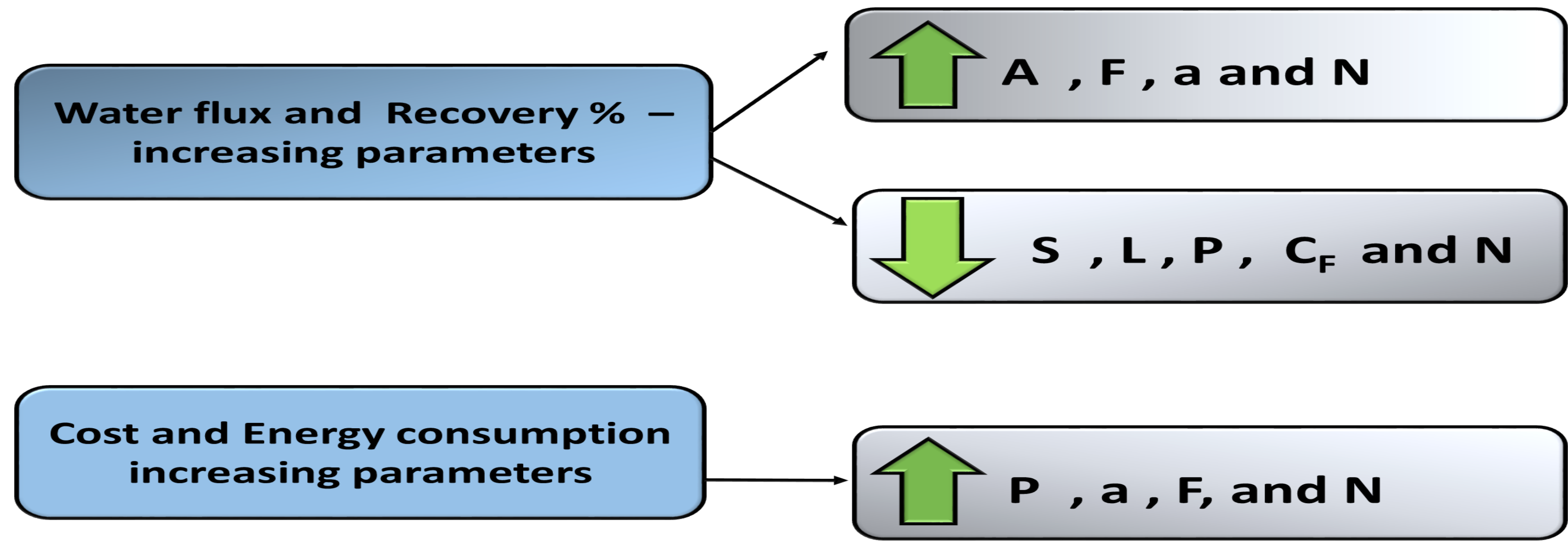

Figure 3. Effect process parameters on OARO performance
[1] Kim, Y. C., \& Min, T. (2020). Influence of osmotic mediation on permeation of water in reverse osmosis: experimental and numerica analysis. Journal of Membrane Science, 595, 117574.

[2] Peters, C. D., \& Hankins, N. P. (2019). Osmotically assisted reverse osmosis (OARO): Five approaches to dewatering saline brines using pressure-driven membrane processes. Desalination, 458, 1-13.

[3] Bartholomew, T. V., Mey, L., Arena, J. T., Siefert, N. S., \& Mauter, M. S. (2017). Osmotically assisted reverse osmosis for high salinity brine treatment. Desalination, 421, 3-11. 